\title{
Giant Transiting Planets Observations with LAIWO
}

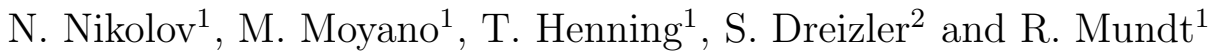 \\ ${ }^{1}$ Max-Planck Institut für Astronomie, Heidelberg, Königstuhl 17, D-69117, \\ Germany [nikolov@mpia.de] \\ ${ }^{2}$ Institut für Astrophysik, Universitët Göttingen, D-370077 Göttingen, Germany
}

\begin{abstract}
We present preliminary single field results on a search for transiting extrasolar planets with the LAIWO wide field optical CCD camera, attached to the 1-m telescope at the Wise Observatory. With a 3 min integration time, the system achieved a few mmag precision for the brightest stars in a field of view of one square degree. We detect several periodic variables and a few transit-like events. The promising candidates will be followed-up in the near future.
\end{abstract}

The Giant Transiting Planets Observations (GITPO) program is a collaboration between the Max-Planck Institut für Astronomie (MPIA), the University of Tel Aviv and the Sternwarte of Göttingen, aiming to detect transiting exoplanets (Afonso et al. 2006). The results in this work are based on single field observations with the Large Area Imager (LAIWO), built at the MPIA for the 1-m telescope at the Wise Observatory, Israel. LAIWO is a wide field optical camera composed by an array of four front-side illuminated $4 \mathrm{~K} \times 4 \mathrm{~K}$ Lockheed CCDs with pixel size of $15 \mu \mathrm{m}$ and scale of $0.87 \mathrm{pixel} / \mathrm{arcsec}$ when binned $2 \times 2$ pixels (Baumeister et al. 2006). Located in Ophiuchus the reported here LAIWO V field was observed $\sim 4000$ times over a total of $\sim 60$ days interval between February 05 and August 06, 2009 and March 02 and June 24, 2010. The images were obtained in the Johnson R-band filter with exposure time set to $180 \mathrm{sec}$. We processed the images using a custom developed automatic IDL pipeline. We considered non saturated stars only, with pixel values $\leq 35,000 \mathrm{ADU}$ to avoid nonlinearity in the CCD. Light curves of $\sim 16,000$ field stars were extracted using aperture photometry with fixed diameter on all images. We identified a set of high quality non-variable reference stars, present on each image, which were used to compute an "average reference star" (ARS), following the method of Scholz \& Eislöffel (2004) to obtain relative magnitudes the light curve of the ARS was subtracted from all time series to correct for atmospheric extinction. We removed systematics with the SysRem algorithm of Tamuz, Mazeh \& Zucker (2005). Root-mean-square (rms) in logarithmic scale versus $\mathrm{R}$ magnitude for all objects is presented in Figure 1. Before the transit search we performed a pre-selection procedure to the collected light curves. We limited our search to stars with photometric accuracy $\leq 20 \mathrm{mmag}$ ( $\mathrm{rms}$ from the entire time series) and standard deviation $\leq 0.02 \mathrm{mag}$. In the next step all remaining stars were searched for transit signal in the period range $\mathrm{P}=1.10$ day to $\mathrm{P}=10.10$ day, using the Box Least Square (BLS) algorithm (Kovacs et al. 2002). BLS detections with signal detection efficiency (SDE) above 3.0 and signal-to-noise ratio $(\mathrm{S} / \mathrm{N})$ greater than 16.0 were selected as significant. To identify the variable stars we applied the Lomb-Scargle algorithm (Lomb 1976) to the rest of the stars. After visual inspection we identified

This is an Open Access article distributed under the terms of the Creative Commons Attribution-Noncommercial License 3.0, which permits unrestricted use, distribution, and reproduction in any noncommercial medium, provided the original work is properly cited. 


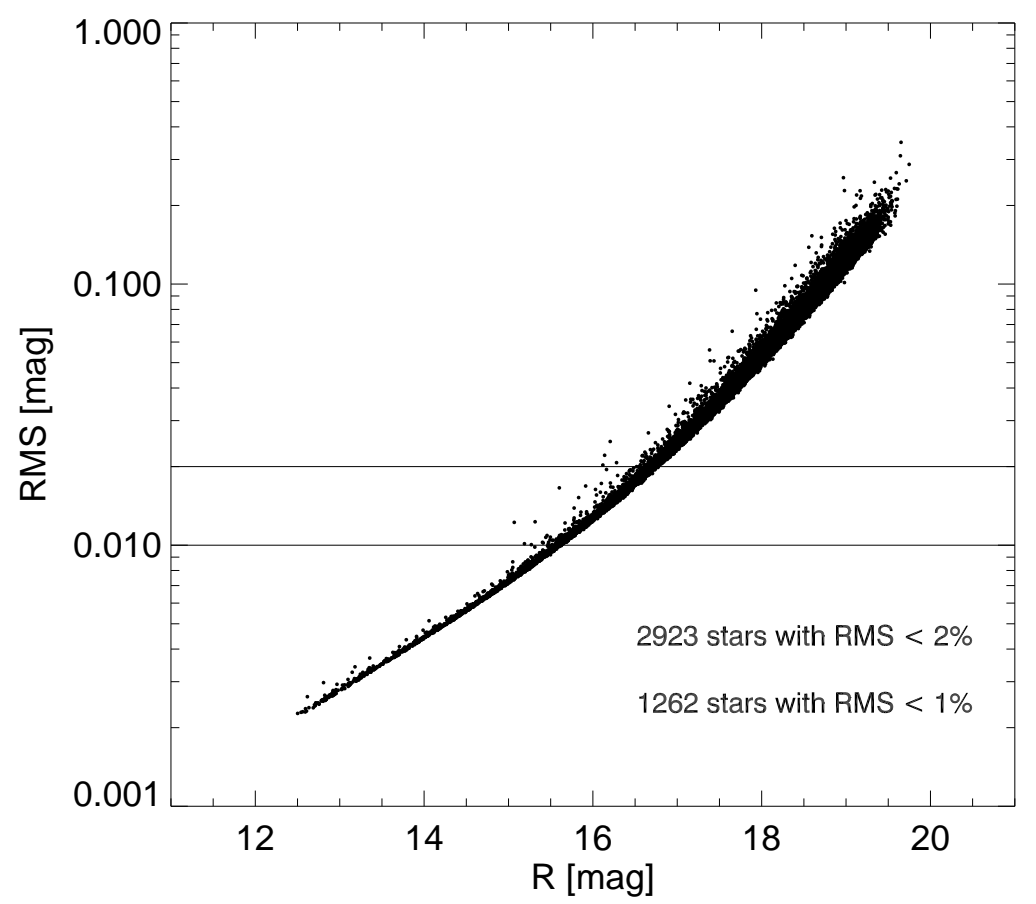

Figure 1: Photometric results for the stars in the LAIWO V field. RMS in logarithmic scale over 20 observations is plotted as a function of the $R$ magnitude, with no detrending applied.

10 periodic variables (with amplitudes 0.2-0.6 mag) and a few transit-like candidates. Figure 2 presents two newly discovered periodic variables and one transit candidate. This and the other candidates will be further analyzed in more detail in the near future.

Acknowledgements. LAIWO, a wide-angle camera operating on the 1-m telescope at the Wise Observatory, Israel, was built at the Max Planck Institute for Astronomy (MPIA) in Heidelberg, Germany, with financial support from the MPIA, and grants from the German Israeli Science Foundation for Research and Development, and from the Israel Science Foundation. The research of N. Nikolov was funded by the Klaus Tschira Foundation (KTF).

\section{References}

Afonso, C., Henning, Th., Weldrake, D., Mazeh, T., \& Dreizler, S. 2006, dies.conf,79A Baumeister, H., Afonso, C., Marien, K.-H., \& Klein, R. 2006, Proceedings of the SPIE, 6269 Kovacs, G., Zucker, S., \& Mazeh, T. 2002, A\&A, 391, 369

Lomb, N. R. 1976, Ap\&SS, 39, 447

Scholz A., \& Eislöffel, J. 2004, A\&A, 419, 249

Tamuz, O., Mazeh, T., \& Zucker, S. 2005, MNRAS, 356, 1466 
Detection and Dynamics of Transiting Exoplanets
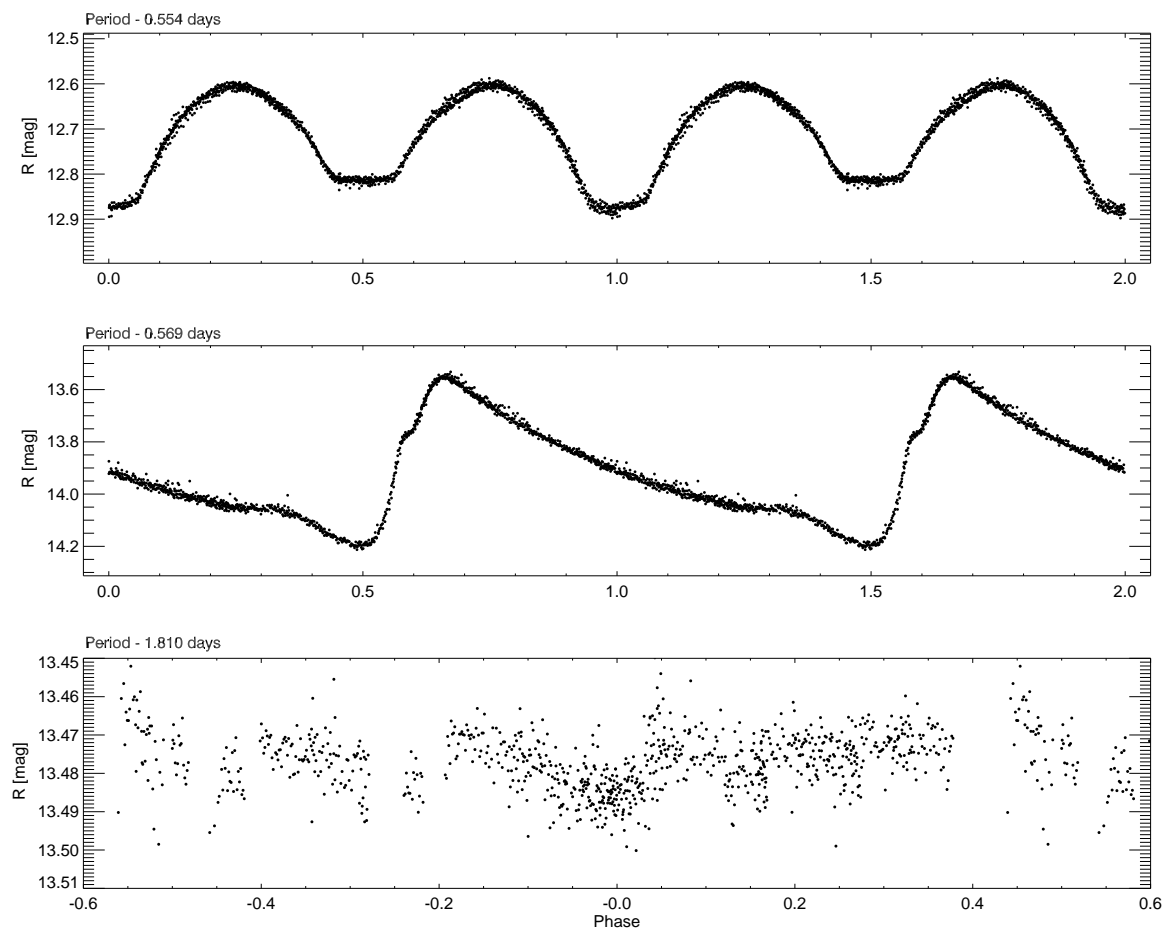

Figure 2: Phase-folded light curves of two newly discovered periodic variables (upper and middle panel) and a transit candidate (bottom panel). Although the third example may not be produced by a planet, it demonstrates the ability of LAIWO and our used reduction software to detect transits. 